\title{
VAC_05 - Designing recombinant MVAs as dual-antigen vector vaccines against SARS-CoV-2
}

Daniel Doro ${ }^{1 *}$; Sabrynna Brito ${ }^{2}$; Alexandre de Magalhães Vieira Machado'; Santuza Maria Ribeiro Teixeira²; Ana Paula Salles Moura Fernandes²; Flávio Guimarães da Fonseca² ${ }^{2}$ Ricardo Tostes Gazzinelli ${ }^{1}$. ${ }^{1}$ Fiocruz/CPqRR;

${ }^{2}$ UFMG - Universidade Federal de Minas Gerais.

Introduction: Recently, the world has witnessed unprecedented agility in vaccine development against COVID-19. To date, only one year since the pandemic outbreak, more than 20 vaccine candidates are currently undergoing large-scale efficacy tests, while at least 10 have been approved for early, limited, or full use around the globe. With the emergence of a number of SARS-CoV-2 variants and as the pharmaceutical companies struggle to supply an ever-increasing demand for doses, the challenge to develop vaccines that will help control the spread of SARS-CoV-2 remains. The Modified Vaccinia virus Ankara (MVA), a well-established viral vector, has been repeatedly shown to be a safe and highly immunogenic system with large capacity to carry heterologous DNA. In fact, recently published studies report successful simultaneous delivery of full-length SARS-CoV-2 Spike (S) and nucleocapsid (N) genes using a recombinant MVA (rMVA). Immunization with this multi-antigenic MVA induced robust antigenspecific humoral and cellular immune responses, as well as neutralizing antibodies.

Objective: Here, we combine RFP and GFP fluorescent reporters in a two-plasmid homologous recombination system to generate MVA-S/N constructs.

Methodology: First, Spike's S1 subunit sequence was cloned into a plasmid containing an early-late mH5 promoter to regulate expression of the heterologous gene, a GFP reporter, under regulation of strong late P11 promoter, and flanking homologous sequences for insertion into the first site of the MVA genome. Secondly, the $\mathrm{N}$ sequence was cloned into a plasmid designed to insert the heterologous gene and the RFP reporter into a second site. The plasmid constructs were then co-transfected with wildtype MVA in Baby Hamster Kidney cells (BHK), whereby homologous recombination occurs. Serial passages into Chicken Embryo Fibroblasts (CEFs) were then performed to replicate the pool of assembled viruses. Using fluorescence-activated cell sorting (FACs) we have selected green (GFP), red (RFP), and yellow (dual insertion) positive cells and lysed them to obtain the rMVAs (MVA-N, MVA-S1, MVA-S1/N).

Results: rMVAs were confirmed using conventional PCR, RT-PCR and Immunoblot.

Conclusion: We have demonstrated an efficient method for obtaining a dual-antigenic MVA to deliver SARS-CoV-2 sequences into mammalian cells. We hope that this will provide alternatives for the vaccine development efforts that our team is currently engaged in.

Keywords: SARS-CoV-2; Viral-based vector vaccines; MVA 